\title{
Prognostic value of systemic inflammatory markers in ovarian Cancer: a PRISMA- compliant meta-analysis and systematic review
}

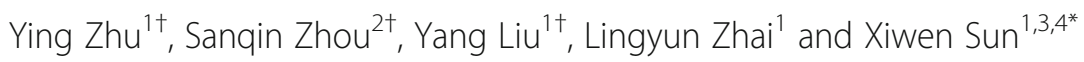

\begin{abstract}
Background: The prognostic effect of elevated systemic inflammatory markers, including neutrophil-lymphocyte ratio (NLR) and platelet-lymphocyte ratio (PLR), remains controversial in cancer patients. This meta-analysis was conducted to evaluate the predictive values of these markers for prognoses in ovarian cancer patients.

Methods: Potentially relevant publications in PubMed, ISI Web of Science, and EBSCO were searched. Pooled hazard ratios (HRs) and corresponding 95\% confidence intervals (95\% Cls) for overall survival (OS) and progressionfree survival (PFS) were determined using a fixed or random effects model.

Results: Ten studies involving 2919 patients were included in this meta-analysis. In multivariate analysis, the group with higher NLR had worse OS ( $H R=1.34,95 \% \mathrm{Cl}=1.16-1.54)$ and shorter PFS ( $H R=1.36,95 \% \mathrm{Cl}=1.17-1.57)$ than the control group. Furthermore, PLR values higher than the cut-off were associated with not only poorer OS (HR $=1.97,95 \%$ $\mathrm{Cl}=1.61-2.40)$ but also more unfavorable PFS ( $\mathrm{HR}=1.79,95 \% \mathrm{Cl}=1.46-2.20)$. Univariate analysis also indicated the same results. Additionally, subgroup analysis showed that when the cut-off values for NLR and PLR were higher, their predictive effects became stronger.

Conclusion: This comprehensive meta-analysis suggested that the values of inflammatory markers such as NLR and PLR were associated with ovarian cancer survival. Therefore, inflammatory markers can potentially serve as prognostic biomarkers.
\end{abstract}

Keywords: Ovarian cancer, Inflammatory markers, Neutrophil-lymphocyte ratio, Platelet-lymphocyte ratio, Prognosis

\section{Background}

Ovarian cancer is a commonly diagnosed gynecologic malignancy and the leading cause of gynecological cancer death in women [1]. Due to the lack of effective screening measures for the early detection of ovarian cancer, nearly $70 \%$ of ovarian cancer patients are diagnosed at an advanced stage. The prognosis of ovarian cancer remains poor; the 5 -year survival rate is approximately $45.6 \%$ overall and approximately $25 \%$ for stage III and IV disease [2].

\footnotetext{
* Correspondence: b1103@zju.edu.cn

${ }^{\dagger}$ Equal contributors

'Department of Gynecology, Second Affiliated Hospital, Zhejiang University School of Medicine, Hangzhou 310009, China

${ }^{3}$ Department of Obstetrics, Second Affiliated Hospital, Zhejiang University

School of Medicine, Hangzhou 310009, China

Full list of author information is available at the end of the article
}

Therefore, the identification of many precise and feasible prognostic factors to guide personalized treatment and predict long-term survival of ovarian cancer patients is urgently needed. Traditional studies focus on the oncological characteristics of the tumor itself (such as tumor histology, grade and gene mutations), but recently, studies have begun to investigate host responses and the tumor microenvironment (such as immune cells, fibrocytes and metabolites) [3]. Systemic inflammatory responses are closely associated with cancer initiation, progression and metastasis, and thus, inflammatory markers, including the neutrophil-lymphocyte ratio (NLR) and platelet-lymphocyte ratio (PLR), have been studied and found to be related to cancer mortality and employed as useful prognostic indicators in many solid tumors [4-6]. A meta-analysis involving 
1540 esophageal cancer patients demonstrated that elevated NLR $(\mathrm{HR}=1.40,95 \% \mathrm{CI}=1.08-1.81)$ and PLR $(\mathrm{HR}=1.59$, $95 \% \mathrm{CI}=1.14-2.21)$ were markedly associated with worse overall survival (OS) and that high NLR also predicted poor OS $(\mathrm{HR}=1.63,95 \% \mathrm{CI}=1.41-1.88)$ for prostate cancer [7, 8]. Additionally, high NLR values before treatment predicted unfavorable OS $(\mathrm{HR}=1.81,95 \%$ $\mathrm{CI}=1.50-2.19)$ and PFS in colorectal cancer $(\mathrm{HR}=2.10$, 95\% CI = 1.55-2.84) [9]. Moreover, Miao and colleagues assessed the predictive value of NLR and PLR in ovarian cancer patients, and the results suggested that patients with lower values of NLR $(\mathrm{NLR}<3.02)$ or PLR (PLR < 207) had longer PFS and OS, and thus, NLR and PLR are significantly related to long-term survival in ovarian cancer [10]. However, a handful of studies demonstrated NLR and PLR as ineffective markers for predicting ovarian cancer prognosis. Raungkaewmanee et al. conducted a retrospective study on 166 epithelial ovarian cancer patients and found that NLR and PLR were not significantly associated with OS or PFS [11]. These contradictory studies thus cannot confirm the predictive effect of inflammatory markers on ovarian cancer survival. Hence, we conducted a systematic review and meta-analysis of published research data to evaluate the predictive value of NLR and PLR in the prognosis for ovarian cancer patients.

\section{Methods}

The present meta-analysis was performed to assess the relationship between pretreatment NLR and PLR and ovarian cancer prognosis according to the protocol of Preferred Reporting Items for Systematic Reviews and Meta-Analysis (PRISMA) [12]. Furthermore, because this study is a meta-analysis without directly involving the handing or inclusion of personal data, ethical approval was not necessary.

\section{Search strategy}

A comprehensive literature search for potentially relevant publications was performed by systematically searching the ISI Web of Science, PubMed and EBSCO using the search terms "neutrophil lymphocyte ratio" (OR "platelet lymphocyte ratio") AND "ovarian cancer" (OR "ovarian carcinoma”) AND "prognosis" (OR "overall survival” OR "progression-free survival"). All databases were searched from January 2000 to April 2017, and the search was updated on November 2017. Bibliographies in each candidate publication were also searched to identify other potentially eligible studies.

\section{Selection criteria}

Two independent investigators carefully screened the candidate publications. Studies were considered eligible if they satisfied all of the following criteria: (i) original articles including patients with histopathologically diagnosed ovarian cancer; (ii) studies providing pretreatment values of relevant inflammatory markers (NLR and PLR) and cut-off values; and (iii) studies reporting the relationship between pretreatment NLR and PLR and prognostic outcomes, with enough data to analyze hazard ratio (HR) and 95\% confidence interval (CI) for PFS or OS. Overlapping or duplicate articles, review articles, letters, case reports, conference abstracts and laboratory studies based on animal models or cancer cell lines were eliminated.

\section{Data extraction and quality assessment}

Information from the included publications was carefully extracted by two independent authors. In addition, any conflict was addressed by joint consensus. The following correlative information was collected in a predefined table from the eligible articles: general information (first author, publication year, and study country), patient characteristics (age, sample size, follow-up duration, cut-off value, and treatment methods) and endpoint parameters (OS and PFS). Many publications showed survival data using the Kaplan-Meier curves, and thus, GetData Graph Digitizer 2.25 (http://getdata-graph-digitizer. $\mathrm{com} /$ ) was used to extract the relevant digitized survival data. Two investigators used the Newcastle-Ottawa-Scale (NOS) to evaluate the quality of the included study, and articles with NOS scores $\geq 6$ were defined as high-quality studies.

\section{Statistical analysis}

Survival outcomes, including OS and PFS, were the primary end-points in this study. Thus, the logarithmic values of HR with $95 \%$ CI were calculated as the primary summary statistic. Heterogeneity among studies was determined using the Cochran Q-test and I-squared test. $\mathrm{I}^{2}>50 \%$ and $p<0.10$ were considered indicators of obvious heterogeneity, and a random effects model was used; otherwise, a fixed effects model was employed. The sources of heterogeneity in the included studies were determined using subgroup analysis and sensitivity analysis. Additionally, funnel plots with Egger's and Begg's tests were adopted for the precise assessment of potential publication bias.

STATA 12.0 software (STATA Corporation, College Station, TX, http://www.stata.com) was employed for all of the statistical analyses, and $p$-values $<0.05$ were considered statistically significant.

\section{Results}

\section{Study characteristics}

A flow diagram representing the study identification procedure is shown in Fig. 1. The initial electronic search strategy yielded 144 potential studies after all duplicated data were removed. Among these, 127 articles were excluded after the screening of titles and abstracts. For further assessment, 17 studies were excluded after full- 


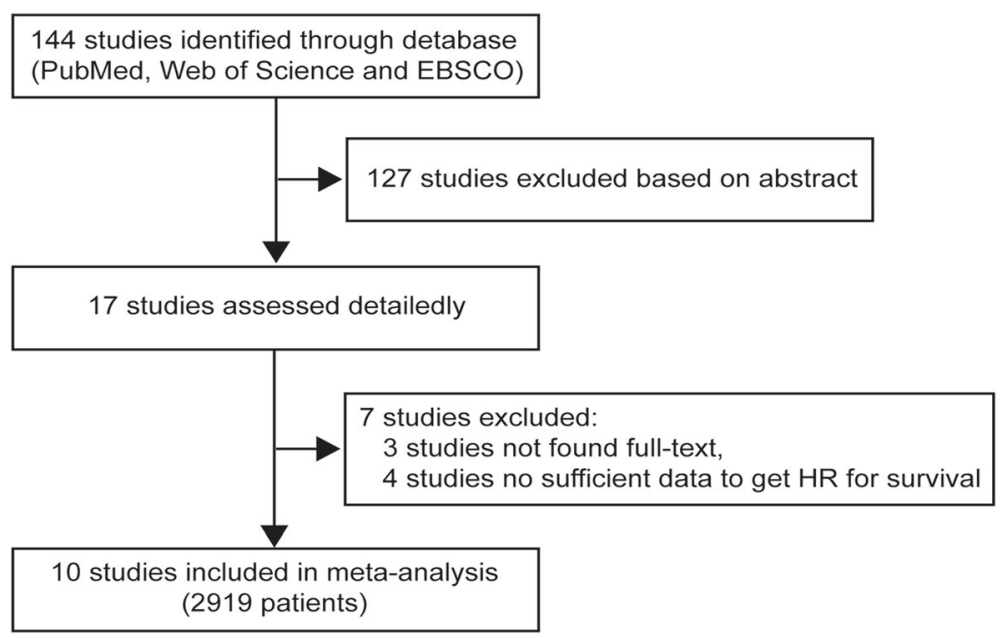

Fig. 1 Flow diagram of study identification

texts were screened. Seven of these studies were excluded. Ultimately, ten retrospective observational cohort studies involving 2919 patients were selected for subsequent meta-analysis [10, 11, 13-20]. The detailed characteristics and quality assessment results of the eligible publications are shown in Table 1. Cut-off values used in the eligible studies for NLR and PLR varied from 187.6 to 300 and 2.6 to 4 , respectively. Additionally, after the evaluation of quality of the included studies, NOS scores of all of the selected articles were $>6$, suggesting high quality.

\section{Association between NLR and ovarian cancer survival}

In univariate analysis, pooled estimates from five datasets revealed that elevated NLR significantly increased the risk of shorter OS (meta-hazard ratio $[$ metaHR] $=2.21,95 \%$ $\mathrm{CI}=1.95-2.52$ ) (Fig. 2a) and shorter PFS (metaHR $=2.22$, 95\% CI $=1.92-2.57$ ) (Fig. 3a). For multivariate analysis, five studies were selected, and the metaHR of $1.34(95 \% \mathrm{CI}=$ 1.16-1.54) indicated that patients with higher NLR have worse OS (Fig. 2b). The synthesized estimates analysis also predicted that the group with elevated NLR had shorter PFS than the control group (metaHR $=1.36,95 \% \mathrm{CI}=$ 1.17-1.57) (Fig. 3b).

\section{Association between PLR and ovarian cancer survival}

Six articles involving 1204 patients calculated hazard ratios of PLR for OS. Combined analysis showed that PLR values higher than the cut-off were associated with a worse OS (metaHR $=2.53,95 \% \mathrm{CI}=2.16-2.96)$ in both univariate and multivariate analyses (metaHR $=1.97$, 95\% CI $=1.61-2.40$ ) (Fig. 4a-b). Pooled analysis of 969 patients included in five studies showed that higher level of PLR indicated unfavorable PFS in both univariate ( metaHR $=2.48,95 \% \mathrm{CI}=2.10-2.96)$ and multivariate analyses $($ metaHR $=1.79,95 \% \mathrm{CI}=1.46-2.20)$ (Fig. 5a-b).

\section{Subgroup analysis}

In some analysis, there was significant heterogeneity in both pooled HR of OS and PFS, and thus, subgroup analyses were conducted to identify the source of heterogeneity. Subgroups were stratified based on cut-off values (NLR: $>3$ vs. $\leq 3$; PLR: $>200$ vs. $\leq 200)$ and sample size (>200 vs. $\leq 200)$ to evaluate HR of OS and PFS. In multivariate analysis, the heterogeneity was noticeable for low cut-off value and small sample size. However, similar results were not obtained using univariate analysis (Tables 2 and 3). Subgroup analysis was also performed according to the subtype of ovarian cancer, the prognostic value of NLR and PLR not only found in whole ovarian cancer, but also in epithelial ovarian cancer (Additional file 1: Table S1).

\section{Publication bias and sensitivity analysis}

Visual inspection of the funnel plot was performed to determine publication bias in the included studies, and the results revealed evident symmetry for NLR and PLR regarding OS and PFS analyses, suggesting the lack of obvious publication bias(Additional file 2: Figure S2). These results were also confirmed using the Begg's and Egger's tests. Furthermore, a sensitivity analysis was performed by assessing the potential impact of each article on pooled HRs. The results showed that no studies had excessive influence on the stability of the pooled effect of comparisons. Therefore, this meta-analysis is robust.

\section{Discussion}

Currently, ovarian cancer survival rates are very variable even among patients with similar disease stage and tumor subtype receiving same therapy. Therefore, prognostic biomarkers are urgently needed to assist the precise prediction of patient prognosis and to facilitate the identification novel therapeutic targets. Many publications have reported 
Zhu et al. BMC Cancer (2018) 18:443

Page 4 of 10

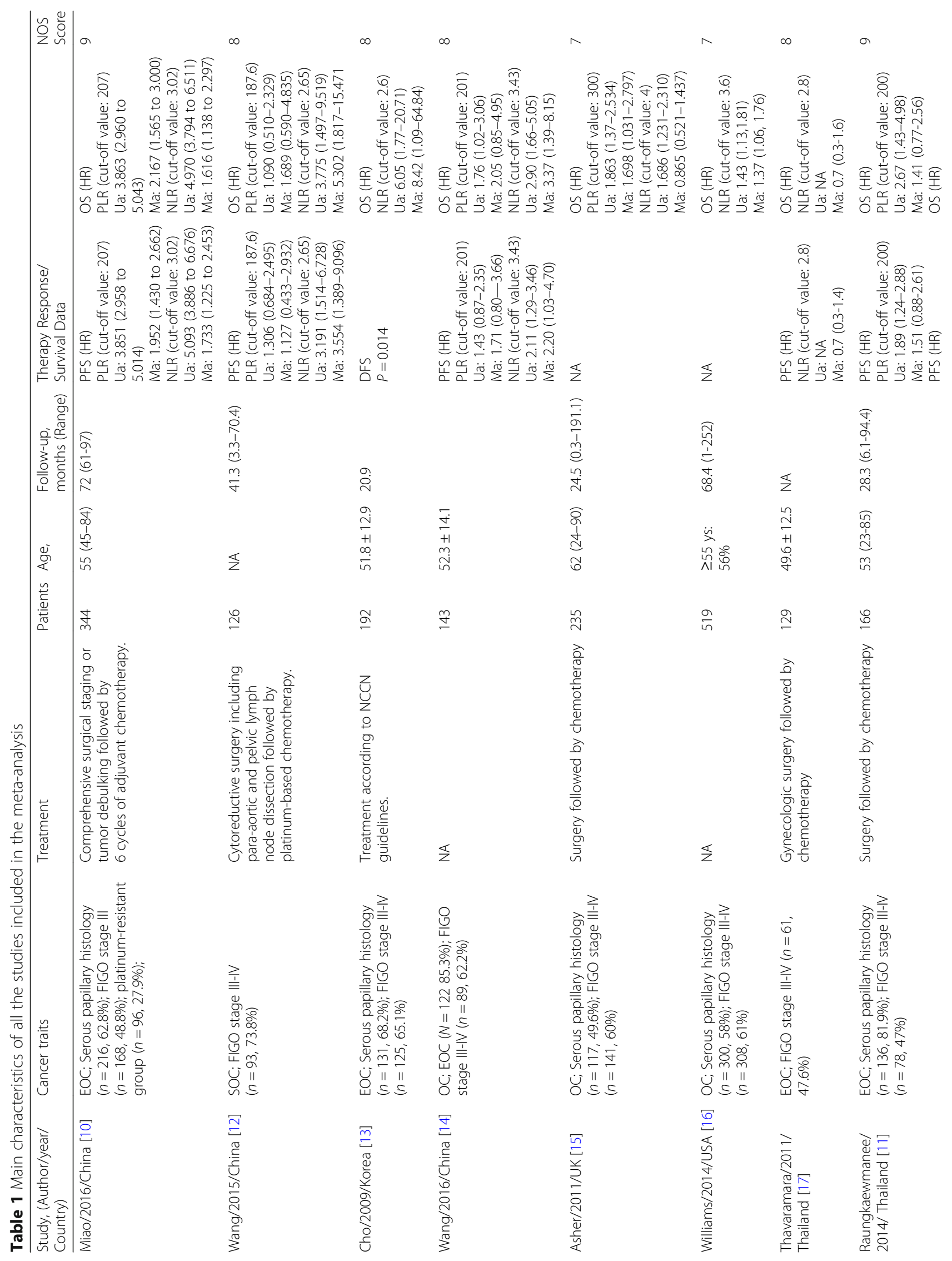




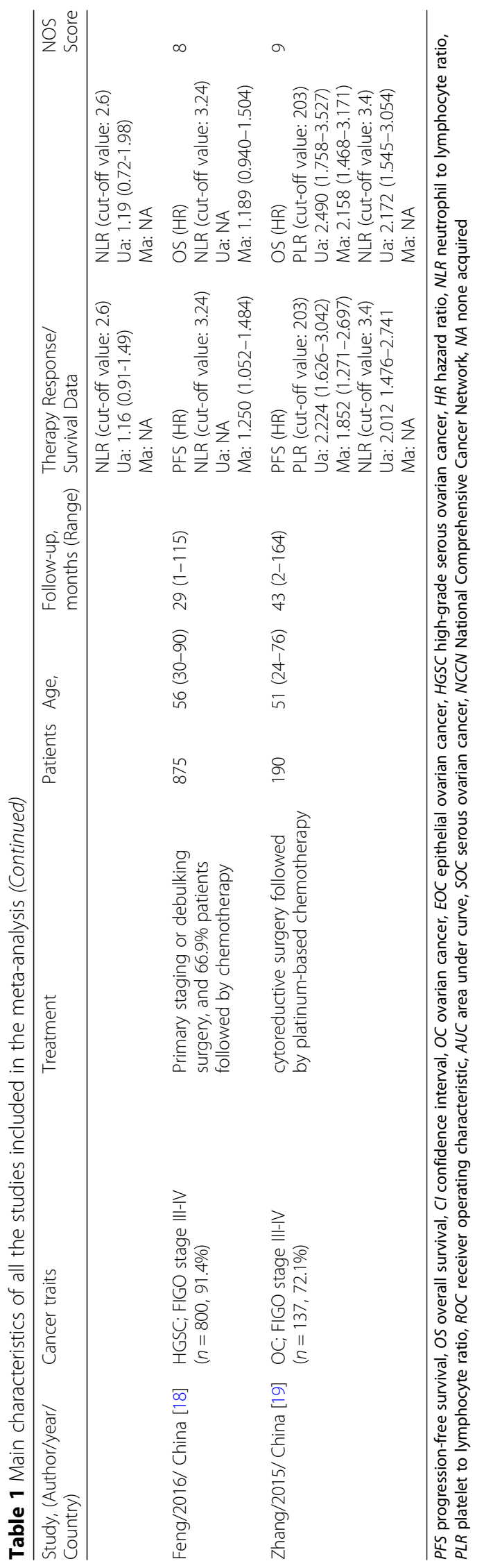




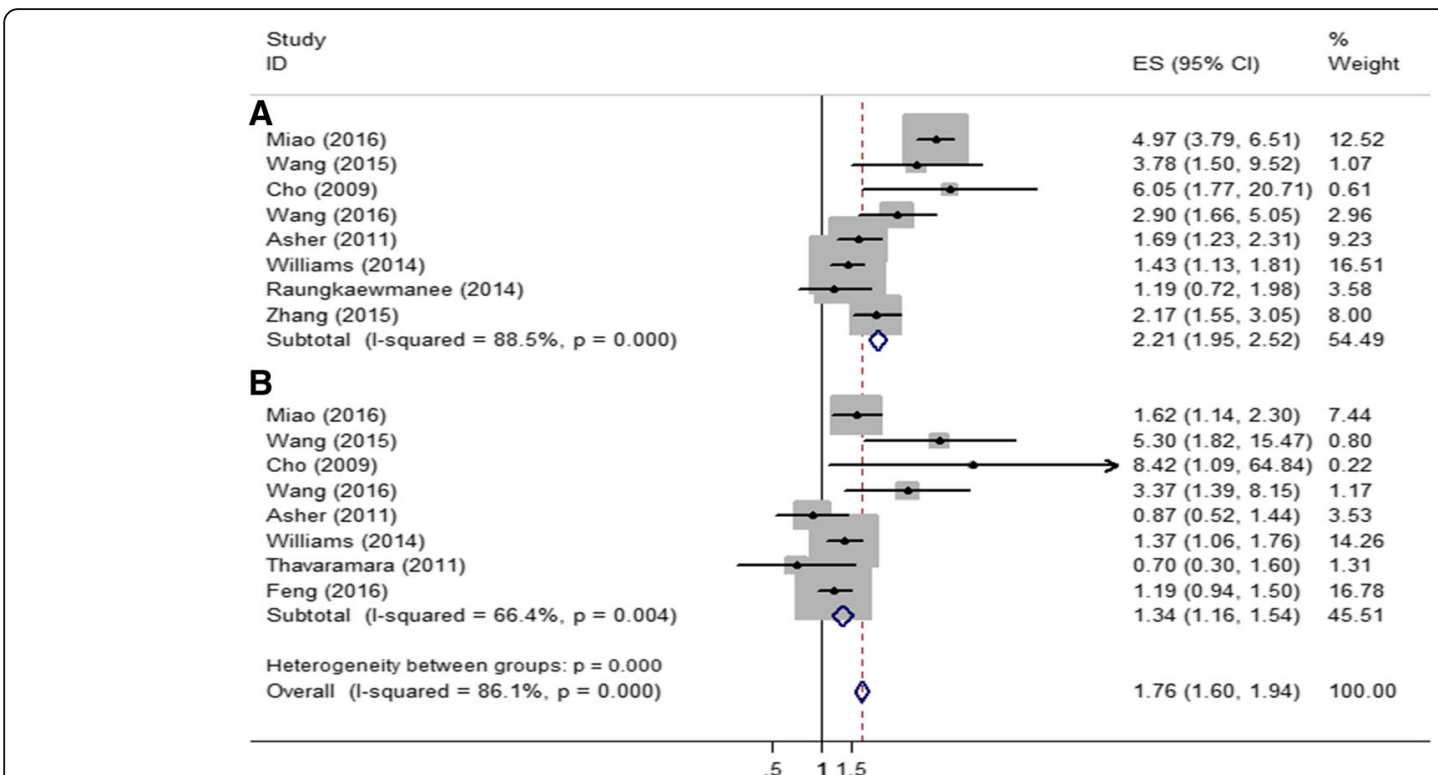

Fig. 2 Forest plots showing the association between NLR and overall survival among ovarian cancer patients. a In multivariate model; (b) In univariate model

the predictive value of inflammatory markers (NLR and PLR) for prognosis in ovarian cancer patients, but the results have been contradictory. This present study is the first meta-analysis assessing the prognostic value of inflammatory markers (NLR and PLR) for long-term survival (OS and PFS) in ovarian cancer patients. Overall, the pooled data provided evidence that both higher NLR and higher PLR predicted inferior survival outcomes. The latest study conducted by Li's group also indicted the predict role [21]. The subgroup analysis also showed that when the cut-off values for NLR and PLR were higher, their predictive effects became stronger.

Many hematological parameters such as neutrophil count, monocyte count, platelet count, NLR and PLR are systemic inflammatory parameters that reflect systemic inflammation and organ dysfunction. NLR, one of markers $\begin{array}{lll}\text { Study } & & \% \\ \text { ID } & \text { ES }(95 \% \mathrm{Cl}) & \text { Weight }\end{array}$

A Miao (2016) Wang (2015) Wang (2016) Raungkaewmanee (2014) Zhang (2015) Subtotal (l-squared $=93.8 \%, p=0.000$ )

B

Miao (2016) Wang (2015) Wang (2016) Thavaramara (2011) Feng (2016)

Subtotal $(1-$ squared $=64.3 \%, p=0.024)$

Heterogeneity between groups: $p=0.000$

Overall ( $(1$-squared $=90.7 \%, p=0.000$ )

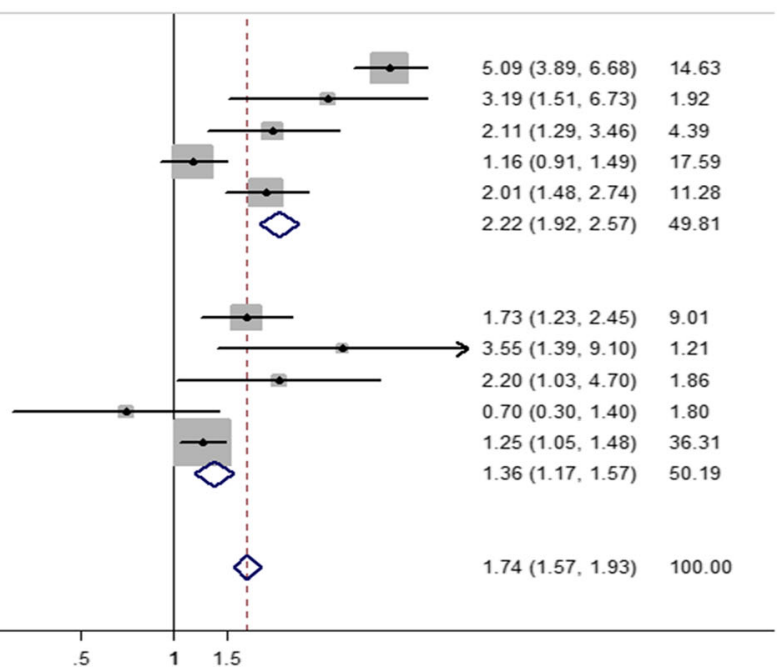

Fig. 3 Forest plots showing the association between NLR and progression-free survival among ovarian cancer patients. a In multivariate model; (b) In univariate model 


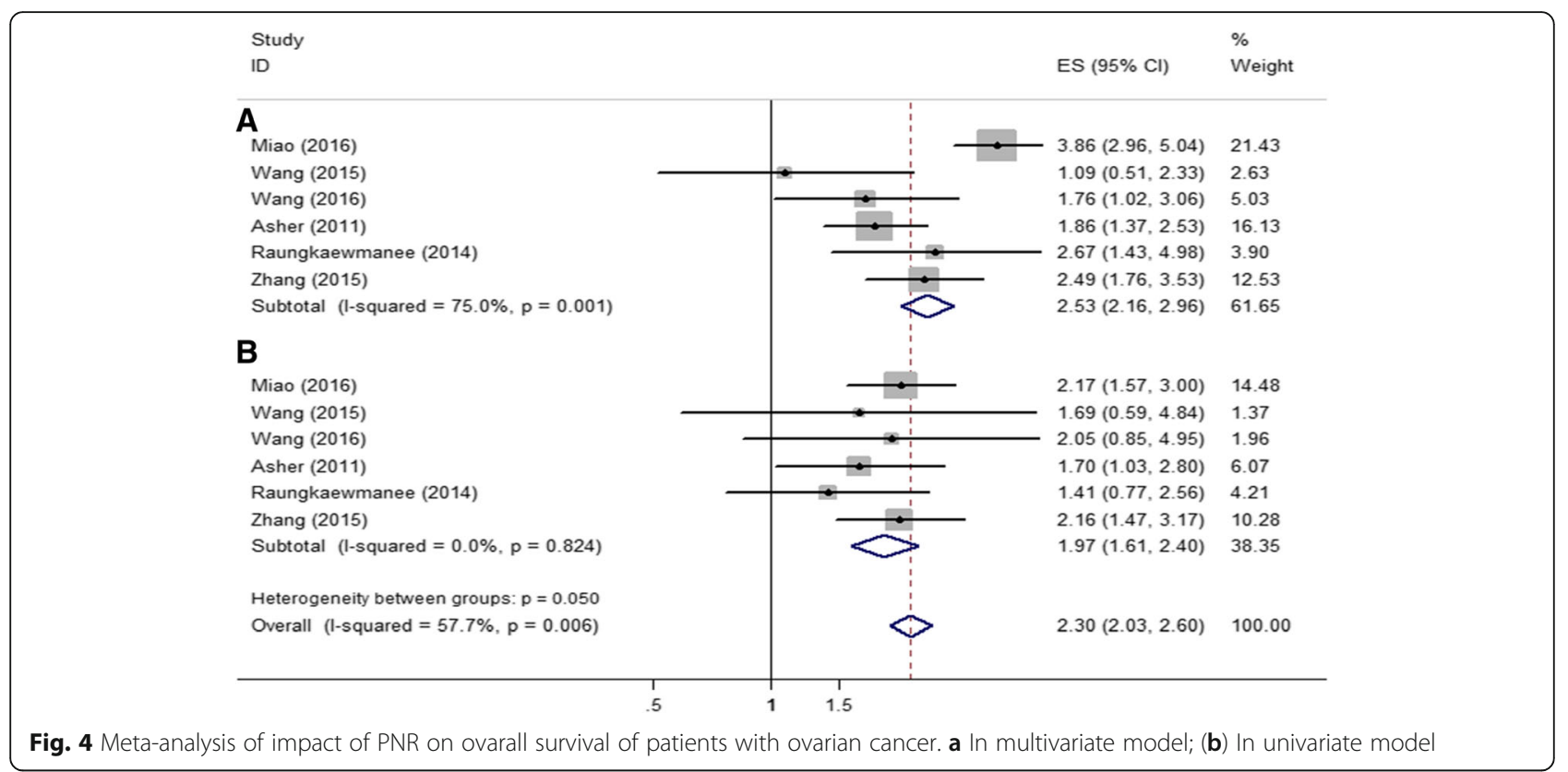

of systemic inflammation, may represent the proangiogenic/pro-inflammatory status in tumor tissues as well as the ratio between neutrophils and lymphocytes, thereby reflecting patients' immune functions. Elevated NLR in patients might indirectly indicate poor lymphocytemediated immune response against tumors, leading to accelerated tumor progression and poor prognosis. Preclinical studies show that neutrophils may act through transforming growth factor $\beta$ (TGF- $\beta$ )-mediated signaling pathways, which can promote tumor cell proliferation [5].
PLR, another indicator with a very important effect on the pathogenesis of systemic inflammatory response, has also been shown to be associated with the prognosis of cancer patients. Platelet counts may be elevated because of the release of inflammatory mediators by tumors or inflammatory cells leading to the stimulation of megakaryocytes to produce platelets. Platelets are also considered to be the major sources of various cytokines, such as vascular endothelial growth factor, that are important for tumor angiogenesis. Clinical studies have indicated NLR and

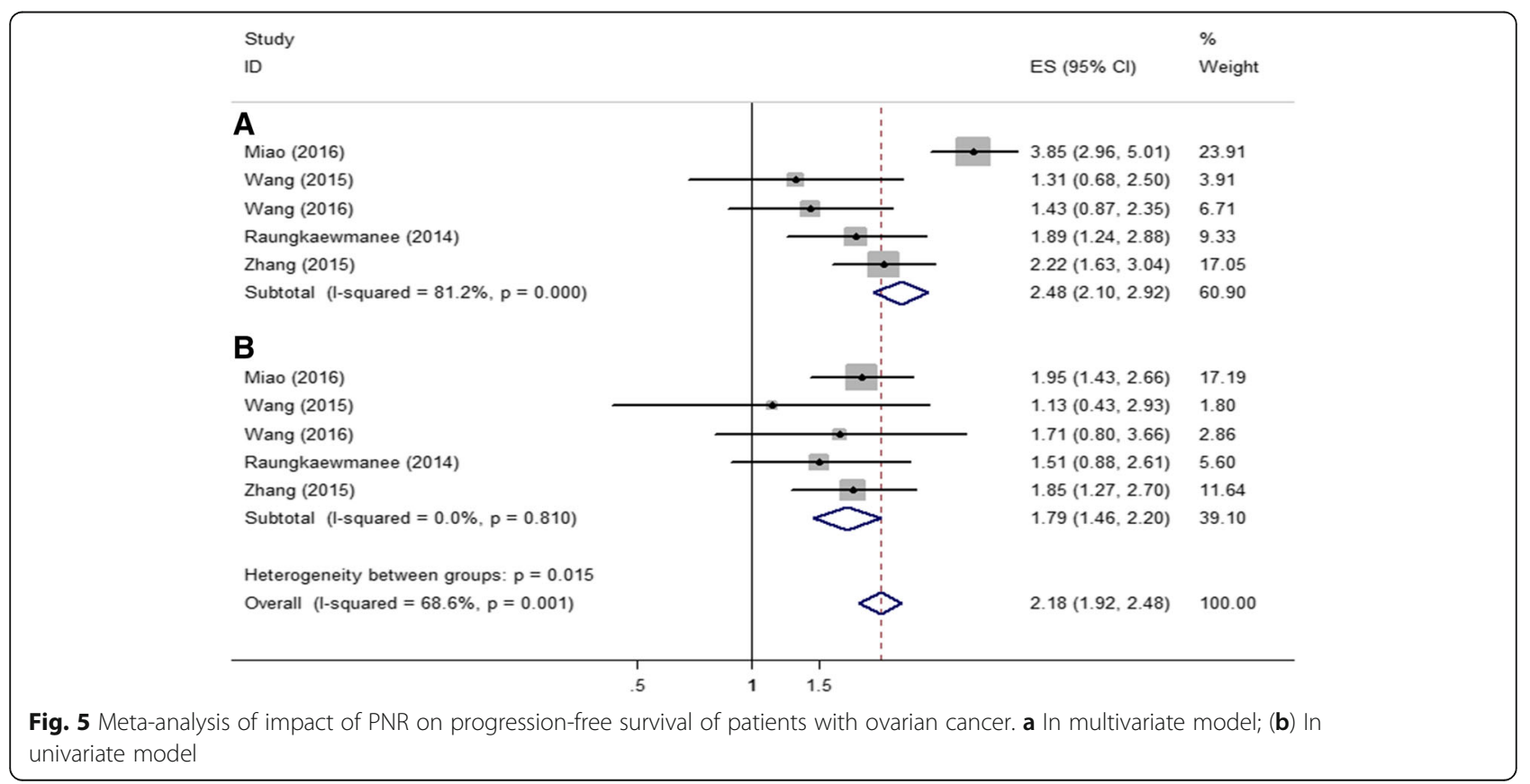


Table 2 Subgroup analysis results of NLR and ovarian cancer survival (OS and PFS)

\begin{tabular}{|c|c|c|c|c|c|c|}
\hline \multirow{2}{*}{$\begin{array}{l}\text { Subgroup } \\
\text { Analysis }\end{array}$} & \multicolumn{2}{|c|}{ Univariate analysis } & \multirow{2}{*}{$\begin{array}{l}\text { Heterogeneity } \\
\text { P1 value }\end{array}$} & \multicolumn{2}{|c|}{ Multivariate analysis } & \multirow{2}{*}{$\begin{array}{l}\text { Heterogeneity } \\
\text { P2 value }\end{array}$} \\
\hline & NO. & ES $(95 \% \mathrm{Cl})$ & & NO. & ES $(95 \% \mathrm{Cl})$ & \\
\hline NLR for OS & 8 & $2.21(1.95-2.52)$ & 0.000 & 8 & $1.34(1.16-1.54)$ & 0.004 \\
\hline \multicolumn{7}{|c|}{ Subgroup 1: cut-off } \\
\hline$>200$ & 5 & $2.26(1.97-2.59)$ & 0.000 & 5 & $1.32(1.14-1.52)$ & 0.060 \\
\hline$\leq 200$ & 3 & $1.82(1.20-2.76)$ & 0.012 & 3 & $1.78(0.95-3.32)$ & 0.004 \\
\hline \multicolumn{7}{|c|}{ Supgroup 2: sample size } \\
\hline$>200$ & 3 & $2.24(1.92-2.61)$ & 0.000 & 3 & $1.35(1.12-1.63)$ & 0.142 \\
\hline$\leq 200$ & 5 & $2.16(1.70-2.74)$ & 0.033 & 5 & $1.32(1.07-1.64)$ & 0.002 \\
\hline NLR for PFS & 5 & $2.22(1.92-2.57)$ & 0.000 & & $1.36(1.17-1.57)$ & 0.024 \\
\hline \multicolumn{7}{|c|}{ Subgroup 1: cut-off } \\
\hline$>200$ & 3 & $3.17(2.63-3.83)$ & 0.000 & & $1.36(1.17-1.58)$ & 0.114 \\
\hline$\leq 200$ & 2 & $1.28(1.01-1.62)$ & 0.012 & & $1.34(0.74-2.44)$ & 0.009 \\
\hline \multicolumn{7}{|c|}{ Supgroup 2: sample size } \\
\hline$>200$ & 1 & 5.09 (3.89-6.68) & / & & $1.33(1.14-1.55)$ & 0.098 \\
\hline$\leq 200$ & 4 & $1.58(1.32-1.88)$ & 0.004 & & $1.62(1.02-2.59)$ & 0.020 \\
\hline
\end{tabular}

PLR as indicators with prognostic implications in many types of cancer, such as breast cancer [22], kidney cancer [23], pancreatic cancer [24], and lung cancer [6]. Studies have also reported that changes in NLR and PLR values are associated with pathological response to neoadjuvant chemotherapy or preoperative chemoradiotherapy in gastric, esophageal and colorectal cancer [25]. Therefore, systemic inflammatory markers have the potential to be used as valuable clinical parameters for predicting chemotherapeutic efficacy and prognosis. It is known that NLR and PLR values can be easily determined from blood tests, and thus, these parameters have great potential for clinical application in the diagnosis and therapy of solid tumors [26].

Nevertheless, this meta-analysis has several limitations. First, the eligible articles described retrospective observational cohort studies, and thus, the evidence was of low quality. Second, this pooled analysis included only ten studies involving 2919 patients, and the small sample size resulted in insufficient data preventing detailed subgroup analysis. Third, although NLR and PLR were easy to measure, the cut-off values were variable and

Table 3 Subgroup analysis results of PLR and ovarian cancer survival (OS and PFS)

\begin{tabular}{|c|c|c|c|c|c|c|}
\hline \multirow{2}{*}{$\begin{array}{l}\text { Subgroup } \\
\text { Analysis }\end{array}$} & \multicolumn{2}{|c|}{ Univariate analysis } & \multirow{2}{*}{$\begin{array}{l}\text { Heterogeneity } \\
\text { P1 value }\end{array}$} & \multicolumn{2}{|c|}{ Multivariate analysis } & \multirow{2}{*}{$\begin{array}{l}\text { Heterogeneity } \\
\text { P2 value }\end{array}$} \\
\hline & NO. & ES $(95 \% \mathrm{Cl})$ & & NO. & ES $(95 \% \mathrm{Cl})$ & \\
\hline PLR for OS & 6 & $2.53(2.16-2.96)$ & 0.001 & 6 & $1.97(1.61-2.40)$ & 0.824 \\
\hline \multicolumn{7}{|c|}{ Subgroup 1: cut-off } \\
\hline$>200$ & 4 & $2.63(2.23-3.10)$ & 0.002 & 4 & $2.06(1.16-2.56)$ & 0.867 \\
\hline$\leq 200$ & 2 & $1.86(1.15-3.01)$ & 0.74 & 2 & $1.47(0.87-2.48)$ & 0.770 \\
\hline \multicolumn{7}{|c|}{ Supgroup 2: sample size } \\
\hline$>200$ & 2 & $2.82(2.31-3.45)$ & 0.000 & 2 & $2.02(1.54-2.65)$ & 0.422 \\
\hline$\leq 200$ & 4 & $2.14(1.67-2.75)$ & 0.193 & 4 & $1.91(1.42-2.55)$ & 0.694 \\
\hline PLR for PFS & 5 & $2.48(2.10-2.92)$ & 0.000 & 5 & $1.79(1.46-2.20)$ & 0.810 \\
\hline \multicolumn{7}{|c|}{ Subgroup 1: cut-off } \\
\hline$>200$ & 3 & $2.75(2.28-3.31)$ & 0.001 & 3 & $1.89(1.50-2.38)$ & 0.943 \\
\hline$\leq 200$ & 2 & $1.70(1.19-2.42)$ & 0.354 & 2 & $1.41(0.88-2.66)$ & 0.606 \\
\hline \multicolumn{7}{|c|}{ Supgroup 2: sample size } \\
\hline$>200$ & 1 & $3.85(2.96-5.01)$ & / & 1 & $1.95(1.43-2.66)$ & / \\
\hline$\leq 200$ & 4 & $1.86(1.51-2.30)$ & 0.330 & 4 & $1.67(1.27-2.20)$ & 0.788 \\
\hline
\end{tabular}


may have been affected by some other conditions, such as bacterial infection and blood coagulation disorders. Therefore, some unexplainable heterogeneity may have existed among the studies.

\section{Conclusion}

In summary, this comprehensive meta-analysis indicated that inflammatory markers such as NLR and PLR are associated with ovarian cancer survival. Therefore, inflammatory markers can potentially serve as prognostic biomarkers. However, to establish the clinical utility of inflammatory markers as prognostic indicators in ovarian cancer, large and well-designed prospective studies are required to confirm clinical significance and to define precise cut-off values for these markers.

\section{Additional files}

Additional file 1: Table S1. Subgroup analysis results of NLR and PLR for ovarian cancer survival (OS and PFS). (DOCX $19 \mathrm{~kb}$ )

Additional file 2: Figure S2. Funnel plot analysis of potential publication bias. (A) NLR for overall survival; (B) NLR for progression-free survival; (C) PLR for overall survival; (D) PLR for progression-free survival. (TIFF $2573 \mathrm{~kb}$ )

\section{Abbreviations}

95\%Cls: 95\% confidence intervals; HRs: Hazard ratios; NLR: Neutrophil-lymphocyte ratio; OS: Overall survival; PFS: Progression-free survival; PLR: Platelet-lymphocyte ratio; PRISMA: Preferred Reporting Items for Systematic Reviews and Meta-Analysis

\section{Acknowledgements}

We thank the useful discussion by our research team and Dr. Zhigang Zhang, at Zhejiang University School of Medicine, for study design and critical review. We would also like to thank the reviewers whose comments and suggestions greatly improved this manuscript.

\section{Funding}

None.

\section{Availability of data and materials}

All data generated or analyzed during this study are included in this published article.

\section{Authors' contributions}

Study design and manuscript writing: $Y Z$ and $X S$. Studies selecting and data analysis: YL, XS and LZ. Study quality evaluating: YL and YZ. Manuscript revising: $S Z$ and $X S$. All authors have read and approved the final manuscript.

\section{Ethics approval and consent to participate}

Not applicable.

\section{Consent for publication \\ Not applicable.}

\section{Competing interests}

The authors declare that they have no competing interests.

\section{Publisher's Note}

Springer Nature remains neutral with regard to jurisdictional claims in published maps and institutional affiliations.

\section{Author details}

'Department of Gynecology, Second Affiliated Hospital, Zhejiang University School of Medicine, Hangzhou 310009, China. ${ }^{2}$ Department of Gynecology,
Changxing people's hospital, Huzhou 313100, China. ${ }^{3}$ Department of Obstetrics, Second Affiliated Hospital, Zhejiang University School of Medicine, Hangzhou 310009, China. ${ }^{4}$ Department of Gynecology and Obstetrics, Second Affiliated Hospital, Zhejiang University School of Medicine, Hangzhou 310009, China.

Received: 26 June 2017 Accepted: 28 March 2018

Published online: 18 April 2018

\section{References}

1. Siegel R, Ma J, Zou Z, Jemal A. Cancer statistics, 2014. CA Cancer J Clin. 2014;64(1):9-29.

2. Matulonis UA, Sood AK, Fallowfield L, Howitt BE, Sehouli J, Karlan BY. Ovarian cancer. Nat Rev Dis Primers. 2016;2:16061.

3. Auer KB-HA, Sukhbaatar N, Aust S, Schmetterer KG, Meier SM, Gerner C, Grimm C, Horvat R, Pils D. Role of the immune system in the peritoneal tumor spread of high grade serous ovarian cancer. Oncotarget. 2016;7(38):61336-54.

4. Orditura M, Galizia G, Diana A, Saccone C, Cobellis L, Ventriglia J, lovino F, Romano C, Morgillo F, Mosca L, Diadema MR, Lieto E, Procaccini E, De Vita F, Ciardiello F. Neutrophil to lymphocyte ratio (NLR) for prediction of distant metastasis-free survival (DMFS) in early breast cancer: a propensity score-matched analysis. ESMO Open. 2016;1(2):e000038.

5. Templeton AJ, McNamara MG, Seruga B, Vera-Badillo FE, Aneja P, Ocana A, Leibowitz-Amit R, Sonpavde G, Knox JJ, Tran B, et al. Prognostic role of neutrophil-to-lymphocyte ratio in solid tumors: a systematic review and meta-analysis. J Natl Cancer Inst. 2014;106(6):dju124.

6. Hong-Bo Yang MX, Lei-Na Ma, Ling-Xin Feng, Zhuang Yu: Prognostic significance of neutrophil-lymphocyteratio/platelet-lymphocyteratioin lung cancers: a meta-analysis. Oncotarget 2016, 2016 Oct 8. https://doi.org/10. 18632/oncotarget.12526.

7. Yodying H, Matsuda A, Miyashita M, Matsumoto S, Sakurazawa N, Yamada M, Uchida E. Prognostic significance of neutrophil-to-lymphocyte ratio and platelet-to-lymphocyte ratio in oncologic outcomes of esophageal Cancer: a systematic review and meta-analysis. Ann Surg Oncol. 2015;23(2):646-54.

8. Sun B, Tang L, Li X, Wang B, Luo G, Gu L, Chen L, Liu K, Gao Y, Zhang X. Prognostic value of neutrophil-to-lymphocyte ratio in localized and advanced prostate Cancer: a systematic review and meta-analysis. PLoS One. 2016;11(4):e0153981.

9. Woo HD, Kim K, Kim J. Association between preoperative C-reactive protein level and colorectal cancer survival: a meta-analysis. Cancer Causes Control. 2015;26(11):1661-70.

10. Miao Y, Yan Q, Li S, Li B, Feng Y. Neutrophil to lymphocyte ratio and platelet to lymphocyte ratio are predictive of chemotherapeutic response and prognosis in epithelial ovarian cancer patients treated with platinum-based chemotherapy. Cancer Biomarkers. 2016;17(1):33-40.

11. Raungkaewmanee S, Tangjitgamol S, Manusirivithaya S, Srijaipracharoen S, Thavaramara T. Platelet to lymphocyte ratio as a prognostic factor for epithelial ovarian cancer. J Gynecol Oncol. 2012;23(4):265.

12. Moher D, Shamseer L, Clarke M, Ghersi D, Liberati A, Petticrew M, Shekelle P, Stewart LA, PRISMA-P Group. Preferred reporting items for systematic review and meta-analysis protocols (PRISMA-P) 2015 statement. Syst Rev. 2015;4(1):2046-4053.

13. Wang Y, Liu P, Xu Y, Zhang W, Tong L, Guo Z, Ni H. Preoperative neutrophil-to-lymphocyte ratio predicts response to first-line platinum-based chemotherapy and prognosis in serous ovarian cancer. Cancer Chemother Pharmacol. 2014;75(2):255-62.

14. Cho H, Hur HW, Kim SW, Kim SH, Kim JH, Kim YT, Lee K. Pre-treatment neutrophil to lymphocyte ratio is elevated in epithelial ovarian cancer and predicts survival after treatment. Cancer Immunol Immunother. 2008;58(1):15-23.

15. Wang YQ, Jin C, Zheng HM, Zhou K, Shi BB, Zhang Q, Zheng FY, Lin F. A novel prognostic inflammation score predicts outcomes in patients with ovarian cancer. Clin Chim Acta. 2016;456:163-9.

16. Asher V, Lee J, Innamaa A, Bali A. Preoperative platelet lymphocyte ratio as an independent prognostic marker in ovarian cancer. Clin Transl Oncol. 2011;13(7):499-503.

17. Williams KA, Labidi-Galy SI, Terry KL, Vitonis AF, Welch WR, Goodman A, Cramer DW. Prognostic significance and predictors of the neutrophil-tolymphocyte ratio in ovarian cancer. Gynecol Oncol. 2014;132(3):542-50.

18. Thavaramara T, Phaloprakarn C, Tangjitgamol S, Manusirivithaya S. Role of neutrophil to lymphocyte ratio as a prognostic indicator for epithelial ovarian cancer. J Med Assoc Thail. 2011;94(7):871-7. 
19. Feng Z, Wen H, Bi R, Ju X, Chen X, Yang W, Wu X. Preoperative neutrophilto-lymphocyte ratio as a predictive and prognostic factor for high-grade serous ovarian Cancer. PLoS One. 2016;11(5):e0156101.

20. Zhang W-w, Liu K-j, Hu G-I, Liang W-j. Preoperative platelet/lymphocyte ratio is a superior prognostic factor compared to other systemic inflammatory response markers in ovarian cancer patients. Tumor Biol. 2015; 36(11):8831-7.

21. Li Z, Hong N, Robertson M, Wang C, Jiang G. Preoperative red cell distribution width and neutrophil-to-lymphocyte ratio predict survival in patients with epithelial ovarian cancer. Sci Rep. 2017;7:43001.

22. Chen Y, Chen K, Xiao X, Nie Y, Qu S, Gong C, Su F, Song E. Pretreatment neutrophil-to-lymphocyte ratio is correlated with response to neoadjuvant chemotherapy as an independent prognostic indicator in breast cancer patients: a retrospective study. BMC Cancer. 2016;16(1):320.

23. Na N, Yao J, Cheng C, Huang Z, Hong L, Li H, Qiu J. Meta-analysis of the efficacy of the pretreatment neutrophil-to-lymphocyte ratio as a predictor of prognosis in renal carcinoma patients receiving tyrosine kinase inhibitors. Oncotarget. 2016;7(28):44039-46.

24. Gemenetzis GBF, Griffin JF, Rezaee N, Javed AA, Manos LL, Lennon AM, Wood LD, Hruban RH, Zheng L, Zaheer A, Fishman EK, Ahuja N, Cameron $J \mathrm{~L}$, Weiss MJ, He J, Wolfgang CL. Neutrophil-to-lymphocyte ratio is a predictive marker for invasive malignancy in Intraductal papillary mucinous neoplasms of the pancreas. Ann Surg. 2017;266(2):339-45.

25. Wu Y, Li C, Zhao J, Yang L, Liu F, Zheng H, Wang Z, Xu Y. Neutrophil-tolymphocyte and platelet-to-lymphocyte ratios predict chemotherapy outcomes and prognosis in patients with colorectal cancer and synchronous liver metastasis. World J Surg Oncol. 2016;14(1):289.

26. Lafrenie RM, Asano Y, Kashiwagi S, Onoda N, Noda S, Kawajiri H, Takashima T, Ohsawa M, Kitagawa S, Hirakawa K. Platelet-lymphocyte ratio as a useful predictor of the therapeutic effect of neoadjuvant chemotherapy in breast Cancer. PLoS One. 2016;11(7):e0153459.

\section{Submit your next manuscript to BioMed Central and we will help you at every step:}

- We accept pre-submission inquiries

- Our selector tool helps you to find the most relevant journal

- We provide round the clock customer support

- Convenient online submission

- Thorough peer review

- Inclusion in PubMed and all major indexing services

- Maximum visibility for your research

Submit your manuscript at www.biomedcentral.com/submit 